\title{
Attosecond photoionization of a coherent superposition of bound and dissociative molecular states: effect of nuclear motion
}

\author{
André D Bandrauk ${ }^{1}$, Szczepan Chelkowski ${ }^{1,5}$, Paul B Corkum ${ }^{2,3}$, \\ Jörn Manz ${ }^{4}$ and Gennady L Yudin ${ }^{1,3}$ \\ ${ }^{1}$ Laboratoire de Chimie Théorique, Faculté des Sciences, Université de Sherbrooke, Sherbrooke, \\ Quebéc J1K 2R1, Canada \\ ${ }^{2}$ University of Ottawa, Ottawa, Ontario K1N 6N5, Canada \\ ${ }^{3}$ National Research Council of Canada, Ottawa, Ontario K1A 0R6, Canada \\ ${ }^{4}$ Institut für Chemie und Biochemie, Freie Universität Berlin, Takustr 3, 14195 Berlin, Germany \\ E-mail: S.Chelkowski@usherbrooke.ca
}

Received 10 January 2009, in final form 5 March 2009

Published 12 June 2009

Online at stacks.iop.org/JPhysB/42/134001

\begin{abstract}
We study numerically the possibility for monitoring electron motion in a dissociating molecule using an attosecond XUV probe pulse which photoionizes a coherent superposition of two nuclear wave packets. We present the photoelectron spectra and forward-backward asymmetries in these spectra obtained from a numerical solution of the time-dependent Schrödinger equation for one electron and two protons both moving in $1 \mathrm{D}$, along the laser polarization vector. In our (non-Born-Oppenheimer) approach the 1D dissociative ionization of the model $\mathrm{H}_{2}^{+}$with softcore potential is described exactly. We find that in general the nuclear motion in a fast moving light molecule does not wash out the oscillations as function of the time delay between XUV probe and the pump pulses as expected from the model with fixed nuclei.
\end{abstract}

(Some figures in this article are in colour only in the electronic version)

\section{Introduction}

In recent years, essential progress has been achieved in attosecond science, see, e.g., [1] for a review and further references. Ultrashort flashes of light allow us to take snapshots of microscopic structures and let us to reconstruct their motion. Recently, pulses as short as 80 as have been obtained [2]. At such timescale monitoring the electron motion becomes in principle possible. A simplest scheme allowing to watch the electron motion inside the atom using two laser pulses $[3,4]$ relies on the preparation of the coherent two bound electronic states (e.g., $1 \mathrm{~s}+2 \mathrm{~s}$ or $1 \mathrm{~s}+2 \mathrm{p}_{0}$ states of the hydrogen atom) followed by an attosecond pulse which photoionizes the atom. Measuring the photoionization signal as function of the time delay between two pulses allows us to 'watch' the oscillating electron.

\footnotetext{
5 Author to whom any correspondence should be addressed.
}

Recently, we have investigated such schemes for monitoring the electron motion in $\mathrm{H}_{2}^{+}$at a fixed internuclear distance $R$ [5-8]. We found that in the case when both states have the opposite parity (like $1 \mathrm{~s}$ and $2 \mathrm{p}_{0}$ states in the hydrogen atom) a particularly convenient way for watching those structures is via the measurement of the photoelectron asymmetries as function of the pump-probe delay time, which is a normalized difference between the photoelectron signal for the momentum $\mathbf{p}$ and $-\mathbf{p}$. This is a most convenient signature of the coherence effect since obviously when the photoionization occurs from a single state the photoelectron angular distribution is symmetric. We found periodic oscillation of asymmetries as function of time delay, see figure 5 in [8], with the oscillation period $T=2 \pi / \omega_{1,2}$ where $\omega_{1,2}$ is the transition frequency between the coherently populated states. These oscillations are synchronized with the changes of probability densities shown in figure 1 in [8] suggesting that the experimental observation of the 
photoelectron asymmetries can be viewed as the observation of the electron motion from one to another centre in a molecule. In order to observe the photoelectron asymmetry one must use a superposition of opposite parity in electron coordinate; there is no asymmetry when both electronic states have the same parity, like $1 \mathrm{~s}+2 \mathrm{~s}$ states in the $\mathrm{H}$ atom, the photoelectron angular distribution is symmetric and in this case one has to rely on the measurement of the total photoionization signal as function of the time delay in order to monitor the timedependent structure as proposed in [3]. The advantages of measurement of photoelectron asymmetries in various contexts were investigated by us earlier [9-12]. Moreover, preparation of the superposition of two states of opposite parity is experimentally simpler since it can be achieved by a resonant one-photon process, whereas in the case of states having the same parity one has to rely on the two-photon process.

All previous theoretical studies (except [13]) of attosecond photoionization of a coherent superposition in molecules were restricted to the molecules fixed at a internuclear distance $R$ and the preparation stage using pump pulse was not included in the dynamics at all. One might expect that nuclear movement will not modify significantly the attosecond ionization process itself since nuclear fragments do not move on the subfemtosecond timescale. However, we expect that the nuclear motion will have an important effect during the preparation of a coherent superposition.

We include the nuclear motion in both stages, i.e. in the preparation of the superposition and of photoionization, by solving numerically the time-dependent Schrödinger equation (TDSE) for a molecular ion $\mathrm{H}_{2}^{+}$in 1D interacting with both the pump and the probe pulses. More specifically, we consider two cases of coherent superpositions, case (I): initial vibrational bound state superposed on a dissociative upper state prepared with a resonant pump pulse and case (II): a superposition of two dissociating wave packets prepared by two pump pulses. We show that the asymmetries in photoelectron spectra in both cases exhibit oscillations as function of the time delay between XUV probe and the pump pulses, as expected from the model with fixed nuclei. In case (I), the oscillations disappear when the dissociating wave packet loses the overlap with the initial state. In case (II), if one achieves the synchronization of the movement of two dissociating overlapping wave packets (using two laser pump pulses), the oscillations persist for much longer time delays than in case (I) despite the fast nuclear motion in the $\mathrm{H}_{2}^{+}$molecule.

\section{Methods and calculations}

For each fixed time delay $t_{\text {del }}$ we have solved numerically the complete, three-body, 1D, TDSE with both electronic and nuclear degrees of freedom included (we use the atomic units (a.u.))

$$
\mathrm{i} \frac{\partial \psi(z, R, t)}{\partial t}=\left[H_{R}(R)+V_{C}(z, R)+H_{z}(z)\right] \psi(z, R, t),
$$

where $z$ is the electron coordinate (with respect to the nuclear centre of mass), $R$ is the internuclear distance, with
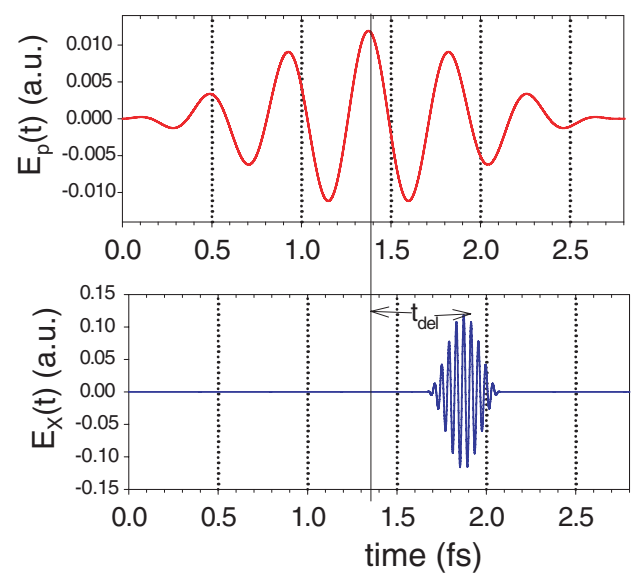

Figure 1. Electric fields of the pump pulse $E_{\mathrm{p}}(t)$ and of the XUV probe pulse $E_{\mathrm{X}}(t)$ used in the excitation scheme (I). The atomic unit (a.u.) of the electric field $=e / 4 \pi \varepsilon_{0} a_{0}^{2}=5.1422 \times 10^{11} \mathrm{~V} \mathrm{~m}^{-1}$, where $a_{0}$ is the Bohr radius.

corresponding electronic $H_{z}(z)$, nuclear $H_{R}(R)$ Hamiltonians,

$H_{z}(z)=-\beta \frac{\partial^{2}}{\partial z^{2}}+\kappa z E(t), \quad H(R)=-\frac{1}{m_{\mathrm{p}}} \frac{\partial^{2}}{\partial R^{2}}+\frac{1}{R}$,

$V_{C}(z, R)=\frac{-1}{\sqrt{(z-R / 2)^{2}+1}}+\frac{-1}{\sqrt{(z+R / 2)^{2}+1}}$,

$\beta=\frac{\left(2 m_{\mathrm{p}}+m_{\mathrm{e}}\right)}{4 m_{\mathrm{p}} m_{\mathrm{e}}}, \quad \kappa=1+\frac{m_{\mathrm{e}}}{2 m_{\mathrm{p}}+m_{\mathrm{e}}}$,

$m_{\mathrm{e}}$ and $m_{\mathrm{p}}$ are respectively the electron and proton masses, $V_{C}$ is the $1 \mathrm{D}$ softcore Coulomb potential. The Hamiltonian used in (1) is the exact three-body Hamiltonian obtained after separation of the centre-of-mass motion [14] in 1D. We have obtained numerically the time evolution of the wavefunction $\psi(z, R, t)$ using the split-operator method [15] and a special wavefunction splitting technique [14], which allows us to recover the probability flux lost in absorbing boundaries, thus allowing us to compute the complete electron kinetic spectra $S(p)$ from the wavefunction $\psi(z, R, t)$ (as described in detail in [14]), where $p$ is the electron momentum. The electric field $E(t)=E_{\mathrm{p}}(t)+E_{\mathrm{X}}(t)$ describes the fields of the pump pulse $E_{\mathrm{p}}(t)$ (or two pump pulses as in case (II)) which prepares a coherent superposition of two electronic states and $E_{\mathrm{X}}(t)$ is the electric field of an attosecond XUV pulse which ionizes this system. The shapes of the laser pulses are defined via the vector potential $A(t)$ (sine-squared envelopes are used), see equations (2) and (3) in [11]. The pulse shapes for case (I) are displayed in figure 1 . We suppose that at $t=0$ the $\mathrm{H}_{2}^{+}$molecule is in its ground state with $v=0$. The pump laser pulse, which is nearly resonant with the transition from the $\sigma_{\mathrm{g}} 1 \mathrm{~s}$ to the $\sigma_{\mathrm{u}} 1 \mathrm{~s}$, prepares a dissociating wave packet on the repulsive surface $\sigma_{\mathrm{u}} 1 \mathrm{~s}$. In case (I), both wave packets are ionized with the help of an attosecond XUV pulse which has the angular frequency $\omega_{\mathrm{X}}=3.675$ a.u. (this corresponds to photon energy equal to $100 \mathrm{eV}$ ). The time delay between these two pulses is defined in figure 1. In case (II), we use a second pump pulse which prepares the second wave packet on the surface $\sigma_{\mathrm{g}} 2 \mathrm{~s}$. The attosecond pulse ionizes then the 


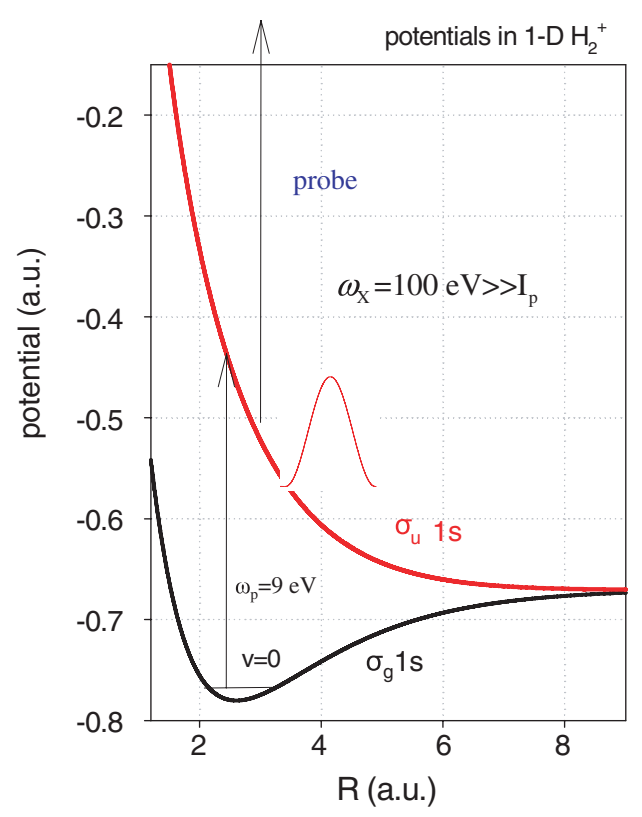

Figure 2. Illustration of the excitation scheme (I) in which two pulses shown in figure 1 are used. The pump pulse prepares the dissociating wave packet on the $\sigma_{u} 1$ s surface which is superposed on the initial $v=0$ nuclear wavefunction which in this scheme remains unchanged. The atomic unit (a.u.) of length is equal to the Bohr radius $a_{0}=0.052918 \mathrm{~nm}$, and the atomic unit of energy on the $y$-axis is $E_{h}=27.211 \mathrm{eV}$.

two dissociating wave packets. In both cases we calculate the electron spectra and asymmetries for a series of selected values of time delays between the probe and the pump pulses (or between the XUV probe pulse and the second pump pulse, in case (II)). The normalized asymmetry coefficient $A_{N}(p)$ for a fixed value of the electron momentum $p>0$ (the case of $p>0$ corresponds to the photoelectron angle $\theta=0$, whereas the case of negative momentum corresponds to $\theta=180^{\circ}$ in the $3 \mathrm{D}$ case) is defined as

$$
A_{N}=\frac{S(p)-S(-p)}{S(p)+S(-p)} .
$$

\section{Case (I): superposition of $\sigma_{\mathrm{g}} 1 \mathrm{~s}+\sigma_{\mathrm{u}} 1 \mathrm{~s}$}

We discuss in this section the photoelectron spectra $S(p)$ and asymmetries obtained from numerical solutions of the TDSE (1) using the pump and probe pulses shown in figure 1. To calculate the spectrum $S(p)$ using the method from [14] we needed to propagate the TDSE (1) until the final $t_{\mathrm{f}}=$ $27 \mathrm{fs}$ for each selected time delay $t_{\mathrm{del}}$. We use the angular frequency of the pump pulse $\omega_{\mathrm{p}}=0.33$ a.u. (the photon energy $-8.98 \mathrm{eV}$ ) which is equal to the energy difference between the $\sigma_{\mathrm{u}} 1 \mathrm{~s}$ and $\sigma_{\mathrm{g}} 1 \mathrm{~s}$, see figure 2 , which illustrates the excitation scheme. The XUV $\left(\omega_{\mathrm{X}}=3.67\right.$ a.u. $)$ attosecond laser pulse is applied next with the time delay $t_{\text {del }}$ defined in figure 1 . The FWHM durations of the pulses, described via sine-squared envelopes, are $\tau_{\mathrm{p}}=1 \mathrm{fs}$ and $\tau_{\mathrm{X}}=0.15 \mathrm{fs}$, for the pump and probe pulses, respectively. The total duration of each pulse is 2.75 time longer than its FWHM duration [11]. Their intensities are $I_{\mathrm{p}}=5 \times 10^{12} \mathrm{~W} \mathrm{~cm}^{-2}$ and $I_{\mathrm{X}}=5 \times 10^{14}$, respectively. Since we expect to see the oscillations in the spectrum with the period $T_{\mathrm{p}}=2 \pi / \omega_{\mathrm{p}}=0.46 \mathrm{fs}$ (see, e.g., figure 5 in [8]) we solved the TDSE for a series of time delays $t_{\text {del }}=0.5 f s+k T_{\mathrm{p}} / 8, k=0,1, \ldots, 11$.

We show in figures 3(a) and 3(c) the photoelectron spectra $S(p)$ for selected time delays obtained from TDSE (1) which includes the nuclear motion. We also show in figures 3(b) and 3(d) the spectra for a static case obtained from TDSE in which the internuclear distance $R$ is fixed at $R=R_{\text {eq }}=$ 2.6 a.u. In all cases shown in figure 3 we observe the change of spectra within time intervals $T_{\mathrm{p}} / 8$, however, we note that the change is smaller in the case when the nuclei move. We checked that for the tritium molecular ion $T_{2}^{+}$which has three times larger reduced mass than $\mathrm{H}_{2}^{+}$the spectra become closer to the static case. However, the difference between these two isotopes is not large in the photoelectron spectra but is more clearly visible in the normalized photoelectron asymmetries $A_{N}\left(t_{\text {del }}\right)$ shown in figure 4 . These asymmetries are calculated (using formula (4)) as a function of time delay for two selected values of the electron momentum, one $p=p_{\max }=2.2$ a.u. corresponds to the maximum of the spectrum $S(p)$, whereas another one, $p=2.4$ a.u., corresponds to the spectrum value three times lower than the value at the maximum. For the static case we expect that these asymmetries should oscillate without attenuation as, e.g., in figure 5 in [8]. The oscillation period seen in figure 4 is indeed only slightly larger than the period $T_{\mathrm{p}}=2 \pi / \omega_{\mathrm{p}}=0.46 \mathrm{fs}$ expected for the static case.

We note that the effect of the nuclear movement is to lower the amplitude of oscillations as function of the time delay $t_{\mathrm{del}}$. Clearly, the oscillations have larger amplitude and survive for a longer time in the $T_{2}^{+}$molecules than in the $\mathrm{H}_{2}^{+}$molecule. This attenuation occurs due to the loss of the overlap between the initial and the dissociating wave packets as seen in figure 5 which shows that the dissociating wave packet in $\mathrm{H}_{2}^{+}$does not overlap with the initial wavefunction already at $t=3 \mathrm{fs}$ which corresponds to the time delay $t_{\mathrm{del}}=1.5 \mathrm{fs}$. Clearly, in the $T_{2}^{+}$molecule this happens 2 fs later than in $\mathrm{H}_{2}^{+}$. Summarizing, measuring the asymmetries in the dissociating molecule allows us to monitor both electronic and nuclear motion. The nuclear motion shows up via attenuation of the oscillation amplitude of the electronic motion.

\section{Case (II): superposition of $\sigma_{\mathrm{u}} 1 \mathrm{~s}+\sigma_{\mathrm{g}} 2 \mathrm{~s}$}

The disappearance of the coherence effect for larger time delays between the probe and the pump pulses seen in the previous section was caused by the loss of the overlap of the dissociating wave packet with the initial $v=0$ nuclear wavefunction. The coherence can be maintained for a longer time in a superposition of two dissociating wave packets which overlap at the moment of their preparation and if they move with similar speed. This can be achieved at larger internuclear distance where molecular surfaces become flat.

To achieve this goal we need two pump pulses; the first one prepares dissociating wave packet on the $\sigma_{\mathrm{u}} 1 \mathrm{~s}$ surface and is followed by a delayed second pump pulse which transfers a part of the population to the upper $\sigma_{\mathrm{g}} 2 \mathrm{~s}$ surface as illustrated 


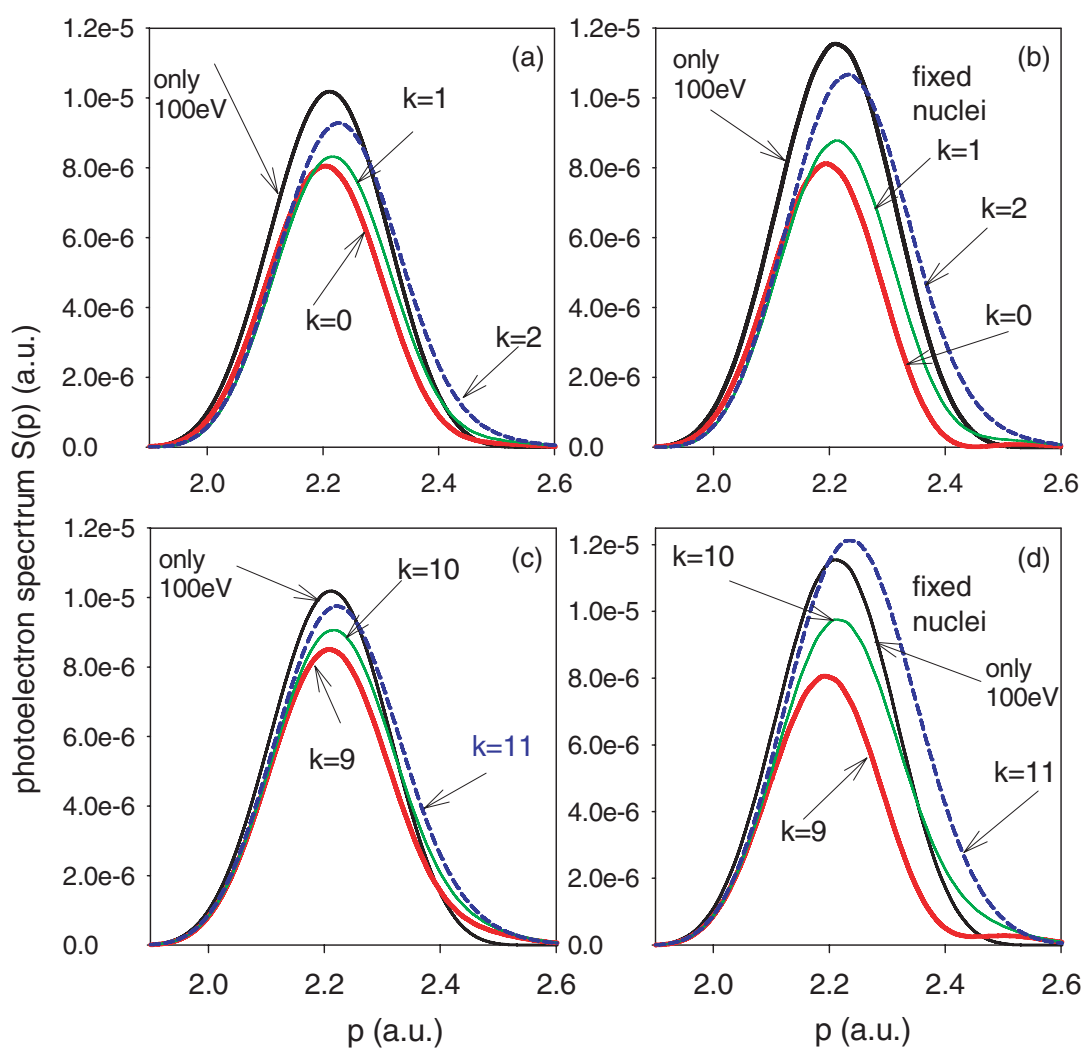

Figure 3. Photoelectron spectra $S(p)$, for case (I), for a series of time delays (defined in figure 1) $t_{\mathrm{del}}=0.5 f s+k T_{\mathrm{p}} / 8, k=0,1, \ldots, 11$, where $T_{\mathrm{p}}=2 \pi / \omega_{\mathrm{p}}=0.46 \mathrm{fs}$ is the period corresponding to the transition frequency between the $\sigma_{\mathrm{g}} 1 \mathrm{~s}$ and $\sigma_{\mathrm{u}} 1 \mathrm{~s}$ surfaces at $R=R_{\mathrm{e}}=$ 2.6 a.u. (a) and (c) show the spectra obtained from TDSE (1) whereas (b) and (d) show spectra from TDSE with fixed internuclear distance $R$. The atomic unit (a.u.) of the momentum is $\hbar / a_{0}$, where $a_{0}$ is the Bohr radius. The atomic unit of the spectrum $S(p)$ is $a_{0} / \hbar$.

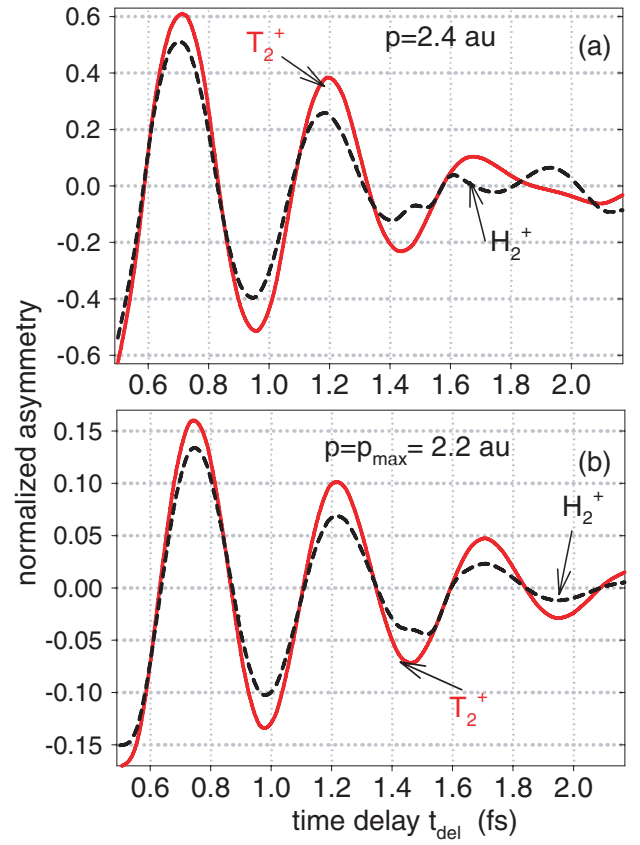

Figure 4. Normalized asymmetries $A_{N}\left(t_{\mathrm{del}}\right)$, for case (I), as function of the time delay $t_{\mathrm{del}}$ calculated at (a) the electron momentum $p=2.4$ a.u. and at (b) $p=p_{\max }=2.2$ a.u.

in figures 6 and 7. The latter figure shows the envelopes of all three pulses used in this excitation scheme. The frequency of the first pump pulse is $\omega_{p 1}=0.228$ a.u. $\left(\lambda_{p 1}=200 \mathrm{~nm}\right.$,

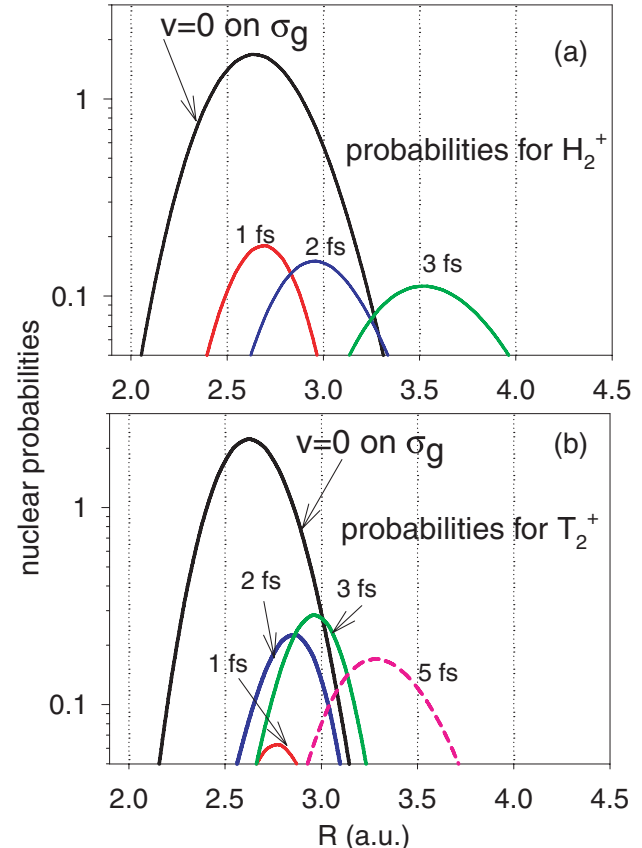

Figure 5. Shapes of the nuclear wave packets dissociating on the repulsive surface $\sigma_{\mathrm{u}} 1 \mathrm{~s}$ calculated at times $t=1,2,3,5 \mathrm{fs}$ compared with the initial $v=0$ state on the $\sigma_{\mathrm{g}} 1 \mathrm{~s}$ surface for (a) a $\mathrm{H}_{2}^{+}$molecule and (b) for a $\mathrm{T}_{2}^{+}$molecule.

photon energy $=6.2 \mathrm{eV}$ ) and of the second is $\omega_{p 2}=0.532 \mathrm{a}$.u. $\left(\lambda_{p 2}=85.6 \mathrm{~nm}\right.$, photon energy $\left.=14.5 \mathrm{eV}\right)$. The latter 


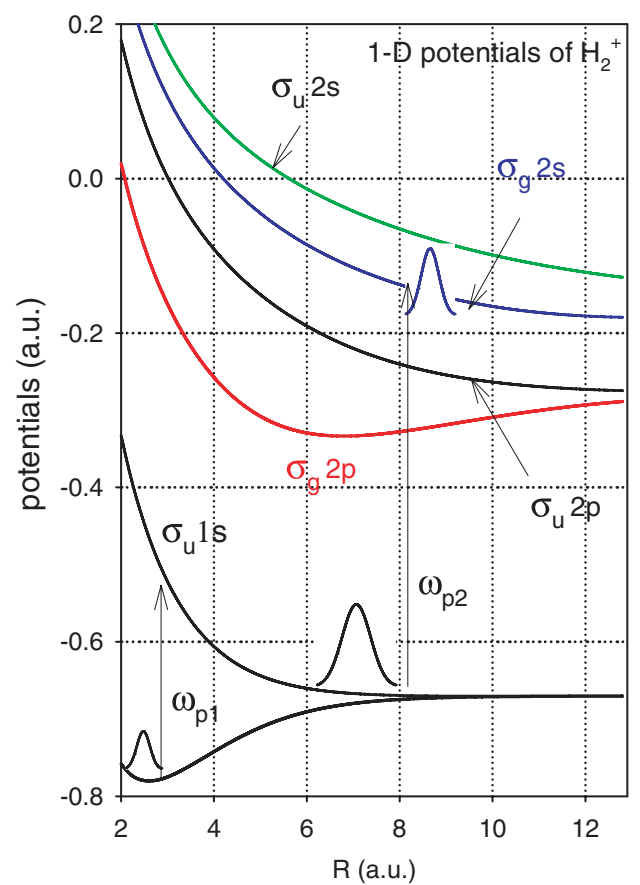

Figure 6. Illustration of the excitation scheme (II) in which two pump pulses, shown in figure 7, are used. First pump pulse (its frequency is $\omega_{p 1}=0.23$ a.u.) prepares a dissociating wave packet on the $\sigma_{u} 1 \mathrm{~s}$ surface. Next, when the dissociating wave packet approaches the internuclear distance $R=8$, a second pump pulse (its frequency is $\omega_{p 2}=0.53$ a.u.) transfers a part of population on upper surface $\sigma_{\mathrm{g}} 2 \mathrm{~s}$. The 1D potentials are found numerically using imaginary time evolution of 1D TDSE without the laser field.

frequency is resonant with the transition frequency from $\sigma_{\mathrm{u}} 1 \mathrm{~s}$ to $\sigma_{\mathrm{g}} 2 \mathrm{~s}$ at $R=8$ a.u. The FWHM durations of the pulses are $\tau_{p 1}=10 \mathrm{fs}, \tau_{p 2}=12 \mathrm{fs}$ and $\tau_{\mathrm{X}}=0.15 \mathrm{fs}$ (as in previous scheme), for the two pump pulses and one probe pulse, respectively. Their intensities are $I_{p 1}=5 \times 10^{13} \mathrm{~W} \mathrm{~cm}^{-2}$, $I_{p 2}=5 \times 10^{13} \mathrm{~W} \mathrm{~cm}^{-2}$ and $I_{\mathrm{X}}=5 \times 10^{14} \mathrm{~W} \mathrm{~cm}^{-2}$ respectively. The total calculation time was $t_{\mathrm{f}}=52 \mathrm{fs}$. We have chosen the time delay between two pump pulses to be $8 \mathrm{fs}$ which allows us to launch a second dissociating wave packet on the upper $\sigma_{\mathrm{g}} 2 \mathrm{~s}$ at $t \simeq 26 \mathrm{fs}$ and thus creating two well-overlapping wave packets at $R>9$ a.u. as seen in figure 8. This figure shows the nuclear probabilities $P_{N}(R, t)$ obtained from the projections of the time-dependent wavefunction $\psi(z, R, t)$ on the Born-Oppenheimer electronic eigenfunctions for the $\sigma_{\mathrm{u}} 1 \mathrm{~s}$ and $\sigma_{\mathrm{g}} 2 \mathrm{~s}$ states.

Figure 9 shows the photoelectron spectra $S(p)$ for selected time delays obtained from TDSE (1). The spectra change significantly within $1 / 8$ of the oscillation period $T_{p 2}=$ $2 \pi / \omega_{p 2}$ expected from a model in which the nuclei would be fixed at $R=8$ a.u. Surprisingly, the ionization signal in the case when only the first pump laser is applied, dotdashed line, is stronger than when two pump pulses are used. Clearly, this is due to the very high intensity of pump pulses we use, $I_{p 1}=I_{p 2}=5 \times 10^{13} \mathrm{~W} \mathrm{~cm}^{-2}$. At this intensities strong ionization may also occur via absorption of 2-5 pump photons. This leads to significantly larger depopulation of the $\sigma_{\mathrm{u}} 1 \mathrm{~s}$ state when two overlapping pump pulses are used, see figure 7 . However, we checked that the peak shown in

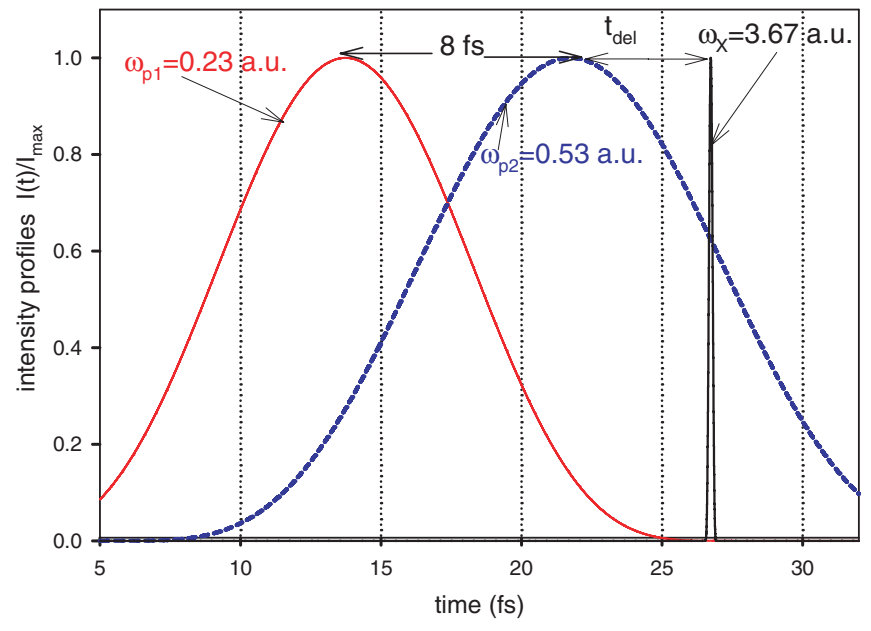

Figure 7. Intensity profiles equal to the pulse envelopes squared (normalized to 1 at the pulse maximum) as function of time used in the excitation scheme (II). The photon energies of the pump pulses and of the probe are $6.2,14.5$ and $100 \mathrm{eV}$, respectively.

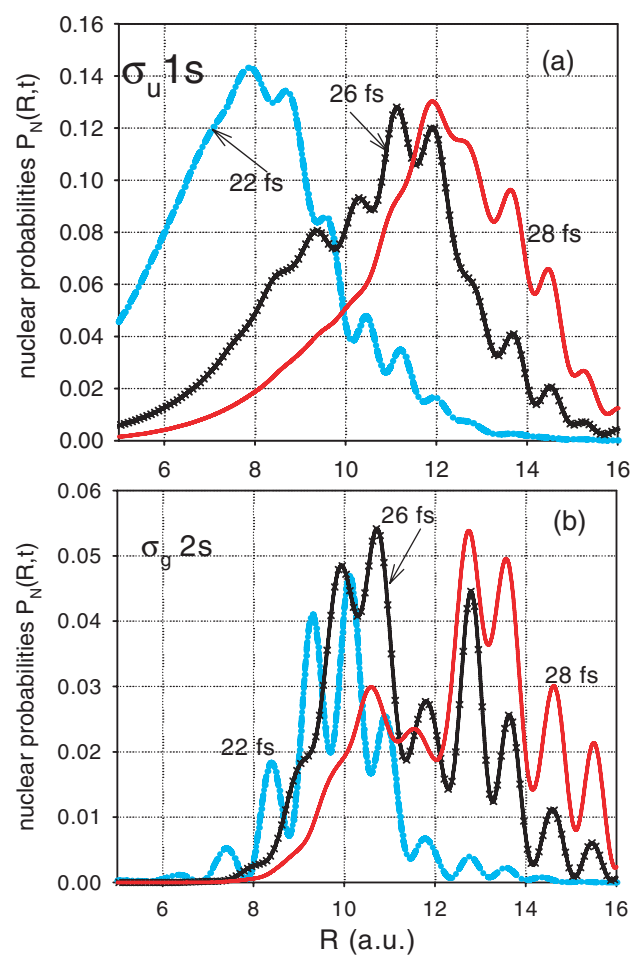

Figure 8. Shapes (probabilities) of the nuclear wave packets dissociating on (a) the lower repulsive surface $\sigma_{\mathrm{u}} 1 \mathrm{~s}$ and on (b) the upper repulsive surface $\sigma_{\mathrm{g}} 2 \mathrm{~s}$, calculated at times $t=22,26$ and 28 fs.

figure 7, which originates from the one-photon absorption, does not overlap with ATI peaks originating from pump pulses.

Finally, we discuss the asymmetries $A_{N}$ as function of the time delay $t_{\text {del }}=5 f s+k T_{p 2} / 8$, shown in figure 10 , which shows the asymmetries as function of the time delay $t_{\text {del }}$ at three selected momentum values. The oscillation period seen in figure 10 agrees very well with the period $T_{p 2}=2 \pi / \omega_{2 p}$ expected from the static case with nuclei fixed at $R=$ 8 a.u. Very small attenuation of the oscillation amplitude is seen. We calculated also the asymmetries for larger time 


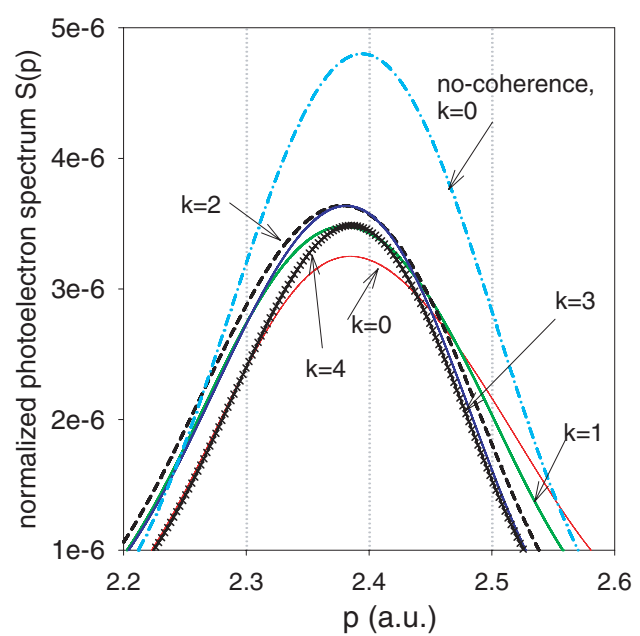

Figure 9. Photoelectron spectra $S(p)$, for case (II)), for a series of time delays $t_{\mathrm{del}}$ with respect to the peak of the second pump pulse, defined in figure 7), $t_{\text {del }}=5 f s+k T_{\mathrm{p}} / 8, k=0,1,2,3,4$. The dashed-dotted line shows the spectrum obtained using only the first pump pulse, i.e. in this case there is no population in the upper state $\sigma_{\mathrm{g}} 2 \mathrm{~s}$. The atomic unit (a.u.) of the momentum is $\hbar / a_{0}$, where $a_{0}$ is the Bohr radius. The atomic unit of the spectrum $S(p)$ is $a_{0} / \hbar$.

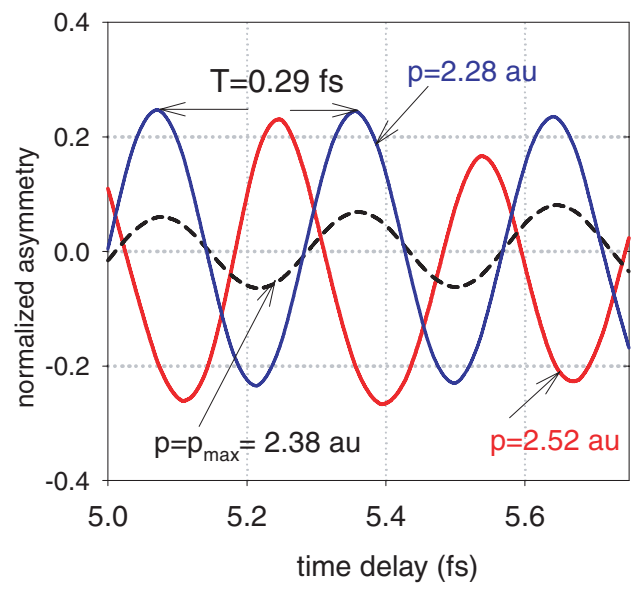

Figure 10. Normalized asymmetries $A_{N}\left(t_{\mathrm{del}}\right)$, for case (II), as function of the time delay $t_{\text {del }}$ calculated at the electron $p=p_{\max }=2.38$ a.u., $p=2.28$ a.u. and $p=2.52$ a.u. The two latter values of momenta correspond to the case when the spectrum is twice weaker than its maximum value, see the case $k=0$ in figure 9.

delays, $t_{\text {del }}>10 \mathrm{fs}$. We display in figure 11 the asymmetries as function of the momentum for a series of time delays: $t_{\text {del }}=10 f s+k T_{p 2} / 8$. We note that the asymmetry does not disappear at larger time delays but shifts to other value of the electron momentum. This effect originates probably from the $\sin (\mathbf{p} \cdot \mathbf{R})$ factor from equation $(15 a)$ in [8]: when the wave packet advances to larger $R$ values the changes occur in asymmetry due to this factor.

\section{Concluding remarks}

In summary, we have extended our previous investigations [68] which were based on a model with a fixed internuclear distance $R$ and in which the preparation process was not included at all in the dynamics but it was assumed ad hoc

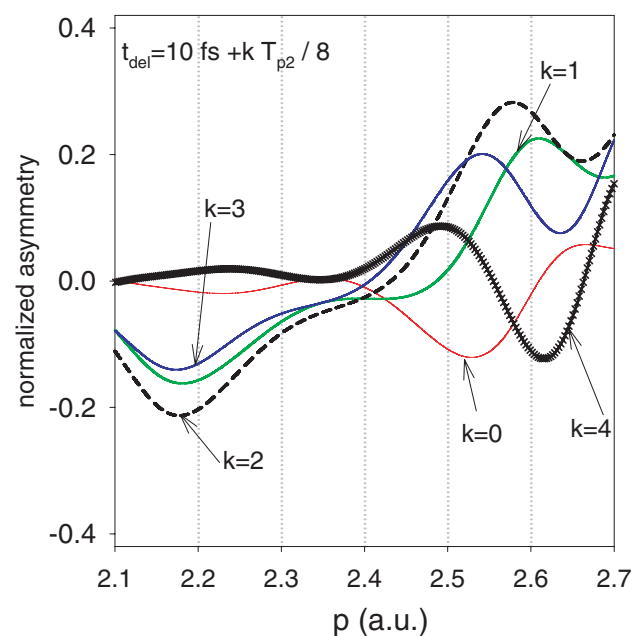

Figure 11. Normalized asymmetries $A_{N}$, for case (II), as function of the electron momentum $p$, calculated for longer time delays than in figure $10, t_{\mathrm{del}}=10 f s+k T_{\mathrm{p}} / 8, k=0,1,2,3,4$. The atomic unit (a.u.) of the momentum is $\hbar / a_{0}$.

that somehow a coherent superposition was prepared. Such an approach was justified for atoms or for heavy molecules but a molecule as light as $\mathrm{H}_{2}^{+}$can clearly move significantly during the preparation process. We have shown that even in light molecules such as $\mathrm{H}_{2}^{+}$the nuclear movement does not destroy the coherence effects seen in the photoelectron spectra as function of the time delay between the probe and the pump pulses. In the case of the superposition of initial (with $v=0$ ) and a dissociative state, case (I), prepared with an ultrashort ( $\tau_{\mathrm{p}}=1 \mathrm{fs}$ ) pump pulse, the narrow dissociating packet is loosing the overlap with the initial $v=0$ nuclear wavefunction leading to the loss of coherence effects in the photoelectron spectra. By contrast, we have found that using longer pump pulses ( $\tau=10,12$ fs, case (II)) allows us to prepare two wider overlapping dissociating superposed wave packets which after ionization using $100 \mathrm{eV}$ photons show the coherence effects expected from the static ( $R$ is fixed) calculations.

\section{Acknowledgments}

ADB and JM thank the Alexander von Humboldt foundation for support through the research award to ADB. Moreover, JM also thanks G K Paramonov (Berlin) for discussions. Financial support by Deutsche Forschungsgemeinschaft and Fonds der chemischen Industrie to JM is also gratefully acknowledged

\section{References}

[1] Corkum P B and Krausz F 2007 Nature Phys. 3381

[2] Goulielmakis E et al 2008 Science 3201614

[3] Krausz F 2001 Phys. World 14 (9) 41

[4] Scrinzi A, Geissler M and Brabec T 2001 Laser Phys. 11169

[5] Bandrauk A D, Chelkowski S and Nguyen H S 2004 Int. J. Quantum Chem. 100834

[6] Yudin G L, Chelkowski S, Itatani J, Bandrauk A D and Corkum P B 2005 Phys. Rev. A 72051401

[7] Yudin G L, Bandrauk A D and Corkum P B 2006 Phys. Rev. Lett. 96063002 
[8] Chelkowski S, Yudin G L and Bandrauk A D 2006 J. Phys. B: At. Mol. Opt. Phys. 39 S409

[9] Bandrauk A D, Chelkowski S and Nguyen H S 2002 Phys. Rev. Lett. 89283903

[10] Bandrauk A D, Chelkowski S and Nguyen H S 2003 Phys. Rev. A 68041802

[11] Chelkowski S and Bandrauk A D 2002 Phys. Rev. A 65061802
[12] Chelkowski S and Bandrauk A D 2005 Phys. Rev. A 71053815 and references therein

[13] Gräfe S, Engel V and Ivanov M Yu 2008 Phys. Rev. Lett. 101103001

[14] Chelkowski S, Foisy C and Bandrauk A D 1998 Phys. Rev. A 571176

[15] Bandrauk A D and Shen H 1993 J. Chem. Phys. 991185 